\title{
UNSTEADY/STEADY NATURAL CONVECTION FLOW OF REACTIVE VISCOUS FLUID IN A VERTICAL ANNULUS
}

\author{
B.K. JHA, A.K. SAMAILA* and A.O. AJIBADE \\ Department of Mathematics, Ahmadu Bello University \\ Zaria, NIGERIA \\ E-mails: basant777@yahoo.co.uk \\ askamba2003@yahoo.co.uk \\ olubadey2k@yahoo.com
}

\begin{abstract}
This paper presents both analytical and numerical analyses of a fully developed unsteady/steady natural convection flow of a reactive viscous fluid in an open ended vertical annulus. Analytical expressions for velocity, temperature, skin-friction and rate of heat transfer are obtained after simplifying and solving the governing differential equations with reasonable approximations. The interesting result found in this study is that an increase in non-dimensional time $(t)$, increases both temperature and velocity profiles until a steady-state value is attained. Subsequent results obtained by numerical calculations show excellent agreement with analytical results.
\end{abstract}

Key words: unsteady/steady natural convection flow, reactive viscous fluid, exothermic, open ended vertical annulus.

\section{Introduction}

An unsteady/steady natural convection flow in a vertical concentric annulus is of interest in several engineering applications such as geothermal energy extraction, oil recovery, food processing, casting and welding of manufacturing process, the dispersion of chemical contaminants in various process in the chemical industry and environment to mention but a few .

The annular geometry is also widely employed in the field of exchangers. A typical example is that of gas-cooled nuclear reactors, in which cylindrical fashionable fuel elements are placed axially in vertical coolant channels within the graphite moderator, the cooling gas flowing along the annulus parallel to the fuel elements.

Unsteady/steady flow formation problems have been studied by many authors, for example: Jha (2001) and Jha et al. (2003) and Singh et al. (1996). Recently, Jha and Ajibade (2010) studied flow formation between vertical parallel plates with one plate isothermally heated and the other thermally insulated. Related works on a steady laminar natural convection in vertical concentric annuli have been studied by El-Shaarawi and Sarhan (1981), El-shaarawi and Al-Nimr (1990) and Hosseini et al. (2005), many studies provided analytical solutions for steady fully developed free-convection flows in vertical annuli considering different thermal configurations. The formulation of reactive viscous fluids was first introduced by Frank-Kamenetskii (1969). Chao et al. (1996) investigated theoretically the heat transfer and reaction characteristics of a chemically reactive forced convection flow near the stagnation point of a catalytic porous bed with finite thickness. A single -reactant, first-order, one-step Arrhenius reaction is assumed to occur. Minto et al. (1998). Merkin and Chaudhary (1994) studied a free-convection boundary-layer flow of a pure, viscous fluid near a stagnation point and along a heated vertical surface. According to these authors there is a three-way coupling between the fluid flow, fluid/surface temperature and reactant species concentration.

\footnotetext{
* To whom correspondence should be addressed
} 
Thus, an interaction between the homogeneous reactions occurring on a catalytic surface is very complex and therefore difficult to model. Makinde $(2005 ; 2006 ; 2008)$ examined exothermic explosions in a heated cylindrical pipe under Biomolecular Arrhenus and sensitized reaction rates neglecting the consumption of the material. Similarly, Ayeni (1982) and Dainton (1960) studied a realistic mathematical desription of thermal expolosion which include the effects of Arrhenius temperature dependence with variable pre-exponential factor. Most recently, Jha et al. (2011) investigated a transient natural convection flow of a reactive viscous flow in a vertical channel formed by two infinite vertical parallel plates. They obtained the transient solution using numerical schemes and the steady-state solution by the perturbation technique. It was pointed out that the rate of heat transfer and skin-friction on both channel plates increase as the reactant consumption parameter increases.

In this study, special attention has been given to the combined effects of Frank-Kamenetskii, activation energy parameters and the Prandtl number on an unsteady/steady natural convection flow of a viscous reactive fluid in a vertical annulus.

\section{Mathematical analysis}

We consider an unsteady fully developed natural convection flow inside a vertical annulus of an infinite length as shown in Fig. 1 immersed in a fluid at a constant temperature $T_{0}$ for $t^{\prime} \leq 0$. At $t^{\prime}>0$, the fluid starts heat generation due to its reactive nature. The fluid is assumed to be Newtonian and obeys Boussinesq's approximation. Under the above assumptions the energy and momentum equations in a dimensional form are

$$
\begin{aligned}
& \frac{d u^{\prime}}{d t^{\prime}}=\frac{v^{\prime}}{r^{\prime}} \frac{d}{d r^{\prime}}\left(r^{\prime} \frac{d u^{\prime}}{d r^{\prime}}\right)+g \beta\left(T^{\prime}-T_{0}\right), \\
& \frac{d T^{\prime}}{d t^{\prime}}=\frac{\kappa}{\rho C p} \frac{1}{r^{\prime}} \frac{d}{d r^{\prime}}\left(r^{\prime} \frac{d T^{\prime}}{d r^{\prime}}\right)+\frac{Q C_{0} A}{\rho C_{p}} \exp \left(-\frac{E}{R T^{\prime}}\right) .
\end{aligned}
$$

The initial and boundary conditions to be satisfied are

$$
\begin{aligned}
& t \leq 0: u^{\prime}=0, \quad T^{\prime}=T_{0} \quad \text { for } \quad a^{*} \leq r^{\prime} \leq b^{*}, \\
& t^{\prime}>0\left\{\begin{array}{llll}
u^{\prime}=0, & T^{\prime}=T_{0} & \text { at } & r^{\prime}=a^{*} \\
u^{\prime}=0 & T^{\prime}=T_{0} & \text { at } & r^{\prime}=b^{*} .
\end{array}\right.
\end{aligned}
$$

It will be useful to obtain the solutions of Eqs (2.1) and (2.2) subject to condition (2.3) in nondimensional forms. To this end, we introduce the non-dimensional quantities

$$
\begin{aligned}
& v=\frac{u^{\prime}}{U_{0}}, \quad t=\frac{t^{\prime} v}{a^{2}}, \quad \theta=\frac{E}{R T_{0}^{2}}\left(T^{\prime}-T_{0}\right), \quad \lambda=\frac{Q C_{0} A E a^{2}}{\kappa R T_{0}^{2}} \exp \left(-\frac{E}{R T_{0}}\right), \\
& \varepsilon=\frac{R T_{0}}{E}, \quad r=\frac{r^{\prime}}{a^{*}}, \quad b=\frac{b^{*}}{a^{*}} .
\end{aligned}
$$


$T^{\prime}$ - dimensional temperature of the fluid, $Q$ - the heat of reaction, $A$ - the rate constant, $E$ - the activation energy, $R$ - the universal gas constant, $C_{0}$ - the initial concentration of the reactant species, $v$ - kinematics viscosity, $r^{\prime}$ - radial co-ordinate, $a^{*}$ - the radius of inner cylinder, $b^{*}$ - the radius of the outer cylinder.

Using expression (2.4) in (2.1) and (2.2) the dimensionless momentum and energy equations are

$$
\begin{aligned}
& \frac{\partial u}{\partial t}=\frac{\partial^{2} u}{\partial r^{2}}+\frac{1}{r} \frac{\partial u}{\partial r}+\theta \\
& \frac{\partial \theta}{\partial t}=\frac{1}{\operatorname{Pr}}\left[\frac{\partial^{2} \theta}{\partial r^{2}}+\frac{1}{r} \frac{\partial \theta}{\partial r}\right]+\frac{\lambda}{\operatorname{Pr}} \exp \left(\frac{\theta}{1+\varepsilon \theta}\right) .
\end{aligned}
$$

While the initial and boundary conditions in dimensionless form are

$$
\begin{gathered}
t \leq 0: \quad u=0, \quad \theta=0 \quad \text { for } \quad 1 \leq r \leq b \\
t>0:\left\{\begin{array}{llc}
u=0, \theta=0 & \text { at } & r=1 \\
u=0, \theta=0 & \text { at } & r=b .
\end{array}\right.
\end{gathered}
$$

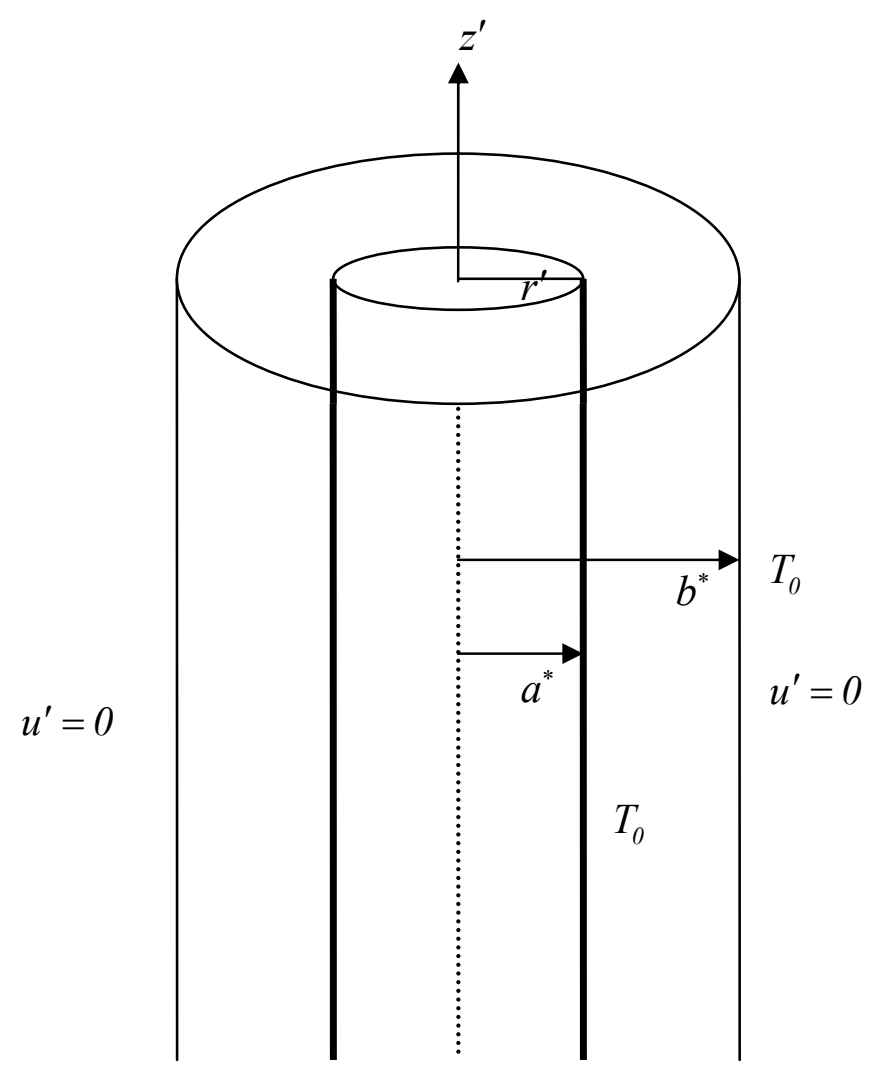

Fig.1. Schematic diagram of the problem. 


\section{Analytical solution}

Analytical solutions for the reactive viscous fluid are obtained for the temperature field and velocity field as well as the skin-friction and Nusselt number. Equation (2.6) is non-linear because of its reactive viscosity it is difficult to find a closed-form solution. Thus perturbation series method is employed. The governing equations of this problem in a closed-form are

$$
\begin{aligned}
& \frac{1}{r} \frac{d}{d r}\left(r \frac{d u}{d r}\right)+\theta=0, \\
& \frac{1}{r} \frac{d}{d r}\left(r \frac{d \theta}{d r}\right)+\lambda \exp \left(\frac{\theta}{1+\varepsilon \theta}\right)=0, \\
& \begin{array}{l}
u=0 \\
u=0
\end{array} \quad \begin{array}{l}
\theta=0 \quad \text { at } \quad r=b .
\end{array}
\end{aligned}
$$
expansion

The solution of Eqs (3.1) and (3.2) subject to Eq.(3.3) can be expressed by the perturbation

$$
\begin{aligned}
& \theta=\sum_{i=0}^{\infty} \theta_{i} \lambda^{i}, \\
& u=\sum_{i=0}^{\infty} u_{i} \lambda^{i} .
\end{aligned}
$$

Substituting Eqs (3.4) and (3.5) into Eqs (3.1) and (3.2) and equating the coefficients of like powers of $\lambda$, the solution of the governing equations are obtained. The solutions for the velocity and temperature fields are

$$
\begin{aligned}
& u=\lambda\left[k_{1} \ln (r)+k_{2}-\frac{r^{2}}{16}+\frac{r^{4}}{64}-\frac{\left(b^{2}-1\right)}{16 \ln (b)}\left(r^{2} \ln (r)-r^{2}\right)\right]+ \\
& +\lambda^{2}\left\{\frac{r^{2}}{256}-\frac{r^{6}}{2304}+\frac{\left(b^{2}-1\right)}{1024 \ln (b)}\left(4 r^{4} \ln (r)-6 r^{4}\right)-\frac{c_{1}}{4}\left(r^{2} \ln (r)-r^{2}\right)-\frac{r^{2}}{4} c_{2}+k_{3} \ln (r)+k_{4}\right\}
\end{aligned}
$$

and 


$$
\begin{aligned}
& \theta=\lambda\left[\frac{1}{4}\left(1-r^{2}\right)+\frac{\left(b^{2}-1\right) \ln (r)}{4 \ln (b)}\right]-\lambda^{2}\left[\frac{r^{2}}{16}-\frac{r^{4}}{64}+\frac{\left(b^{2}-1\right)}{16 \ln (b)}\left(r^{2} \ln (r)-r^{2}\right)-c_{1} \ln (r)-c_{2}\right]+ \\
& +\lambda^{3}\left\{\frac{r^{4}}{256}-\frac{r^{6}}{2304}+\frac{\left(b^{2}-1\right)}{4 \ln (b)}\left(\frac{r^{4} \ln (r)}{64}-\frac{6 r^{4}}{256}\right)-\frac{c_{1}}{4}\left(r^{2} \ln (r)-r^{2}\right)-\frac{r^{2}}{4} c_{2}+c_{3} \ln (r)+c_{4}+\right. \\
& +\left(\frac{2 \varepsilon-1}{4}\right)\left(\frac{1}{1152}\left(18 r^{2}-9 r^{4}+2 r^{6}\right)+\frac{\left(b^{2}-1\right)}{256 \ln (b)}\left(8 r^{2} \ln (r)-2 r^{4} \ln (r)-8 r^{2}+r^{4}+\right.\right. \\
& \left.+\frac{\left(b^{2}-1\right)^{2}}{128[\ln (b)]^{2}}\left(2 r^{2}[\ln (r)]^{2}-4 r^{2} \ln (r)+3 r^{2}\right)\right\} .
\end{aligned}
$$

Steady-state skin frictions on the boundaries are

$$
\begin{aligned}
& \tau_{l}=\left.\frac{d u}{d r}\right|_{r=1}=\lambda\left[k_{1}-\frac{1}{16}+\frac{\left(b^{2}-1\right)}{16 \ln (b)}\right]+\lambda^{2}\left[\frac{5}{384}-\frac{5\left(b^{2}-1\right)}{256 \ln (b)}+\frac{1}{4}\left(c_{1}-2 c_{2}\right)+k_{3}\right], \\
& \tau_{b}=\lambda\left[\frac{b^{3}}{16}-\frac{b}{8}-\frac{\left(b^{2}-1\right)}{4 \ln (b)}\left(-\frac{4}{b}+\frac{b}{2} \ln (b)+\frac{k_{1}}{b}\right)\right]+ \\
& +\lambda^{2}\left[\frac{b^{3}}{64}-\frac{b^{5}}{384}+\frac{\left(b^{2}-1\right)}{4 \ln (b)}\left(\frac{b^{3} \ln (b)}{16}-5 \frac{b^{3}}{64}\right)-c_{1}\left(\frac{b \ln (b)}{2}-\frac{b}{4}\right)-\frac{b}{2} c_{2}+\frac{k_{3}}{b}\right] .
\end{aligned}
$$

Due to the symmetric condition of the flow, the heat transfer on both boundaries is the same and therefore, the steady-state rate of heat transfer on the boundary plate is

$$
\begin{aligned}
& \mathrm{Nu}=\frac{d \theta}{d y}=\lambda\left[\frac{1}{b} \frac{\left(b^{2}-1\right)}{4 \ln (b)}-\frac{b}{2}\right]-\lambda^{2}\left[\frac{b}{8}-\frac{b^{3}}{8}+\frac{\left(b^{2}-1\right)}{16 \ln (b)}\left(\frac{b}{2}+2 b \ln (b)\right)-\frac{c_{1}}{b}\right]+ \\
& +\lambda^{3}\left\{\frac{b^{4}}{256}-\frac{b^{6}}{2304}+\frac{\left(b^{2}-1\right)}{4 \ln (b)}\left(\frac{b^{4} \ln (b)}{64}-\frac{6 b^{4}}{256}\right)-\frac{c_{1}}{4}\left(b^{2} \ln (b)-b^{2}\right)-\frac{b^{2} c_{2}}{4}+c_{1} \ln (b)+\right. \\
& +c_{2}+\left(\frac{2 \varepsilon-1}{4}\right)\left[\frac{b^{2}}{4}-\frac{b^{4}}{128}+\frac{b^{6}}{576}+\frac{\left(b^{2}-1\right)}{8 \ln (b)}\left(\frac{b^{2} \ln (b)}{4}-\frac{b^{4} \ln (b)}{16}-\frac{b^{2}}{4}+\frac{b^{4}}{32}\right)+\right. \\
& \left.\left.+\frac{\left(b^{2}-1\right)^{2}}{16[\ln (b)]^{2}}\left(\frac{b^{2}[\ln (b)]^{2}}{4}-\frac{b^{2} \ln (b)}{2}+\frac{3 b^{2}}{8}\right)\right]\right\}
\end{aligned}
$$

where, $c_{1}, c_{2}, c_{3}, c_{4}$ are defined in the appendix. 


\section{Numerical solutions}

The governing differential equations have been transformed into the corresponding finite difference equations. The implicit finite difference equations are derived using a second-order central difference scheme for the spatial derivatives. The convergence analysis of the present numerical scheme has been performed, and it is found that the derived finite difference equations resemble a consistence representation of the analytical solution. In each time step, firstly the temperature field has been solved and then the velocity field was evaluated using the already known values of the temperature field and the process of computation was continued until a steady state was reached. The analytical solution was employed as a check on the accuracy and effectiveness of the numerical solution.

\section{Results and discussion}

For the numerical validation of our results, we have chosen physically meaningful values of the parameters entering the problem. The annulus model is more general than the model cylindrical pipe or two parallel planes. When $\lambda \rightarrow \infty$, the annulus approaches a cylindrical pipe. The effect associated with the outer cylinder can be ignored. On the other hand, as $\lambda \rightarrow 1$, the annulus behaves like two parallel plates and therefore both cylinders play equally important roles. When the value of $\lambda$ is in between, the curvature differences between the inner and outer walls can lead to quite different behaviors. Also, it is important to note that $\lambda$ is essentially the viscous heating parameter. Typical variations of the fluid temperature profiles are shown in Fig. 2 corresponding to air $(\operatorname{Pr}=0.71)$ and $(\lambda=0.1,0.2,0.3)$. It is seen from the figure that the temperature increases with time and ultimately reaches its steady-state value. It is also seen that as the value of the viscous heating parameter $(\lambda)$ increases the temperature increases until the steady-state value is reached. In Fig.3, the velocity profile is shown for an increasing value of $(\lambda)$ and $t(\operatorname{Pr}=0.71)$. Generally, the fluid velocity increases with increasing values of $t$ until they attain their steady-state values. Also, increasing $\lambda$ increases velocity. This is because increasing the viscous heating parameter means that heating is increased and density is reduced and the buoyancy effect is increased and as a result velocity increases. The variation of the skin-friction at the plates is shown in Figs 4 and 5 for different values of $t$ corresponding to the isothermal boundary $(r=1)$ and $(r=b)$ for $(\operatorname{Pr}=0.71, \varepsilon=0.01, \lambda=0.1,0.2,0.3)$. It is clear from these figures that the skin-friction is directly proportional to time and finally attains its steady-state value. It is seen in Fig.5 that the steady-state is reached earlier as compared to Fig.4. This is because the friction is more on the isothermal boundary than at $(r=b)$. Figure 6 shows how the Nusselt number changes with time. It follows from the figure that the rate of heat transfer is directly proportional to the non-dimensional time. Also, an increase in $\lambda$ has the tendency to warm up the fluid and to reduce the heat transfer on the boundary. The skin-friction and Nusselt numbers are calculated using numerical simulation and these numerical values are compared with the steady-state values obtained. 


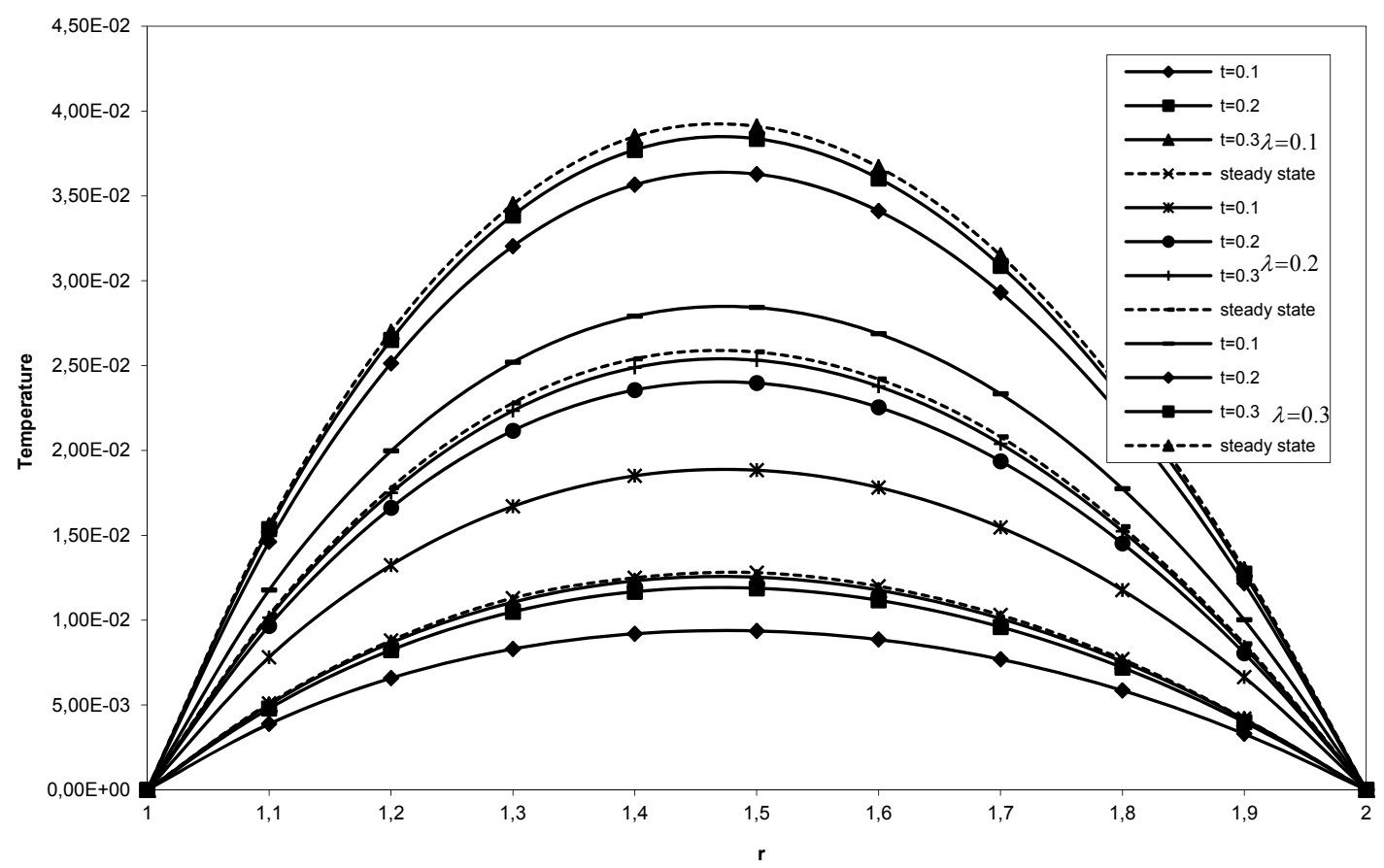

Fig.2. Temperature profile $(\varepsilon=0.01, \operatorname{Pr}=0.71)$.

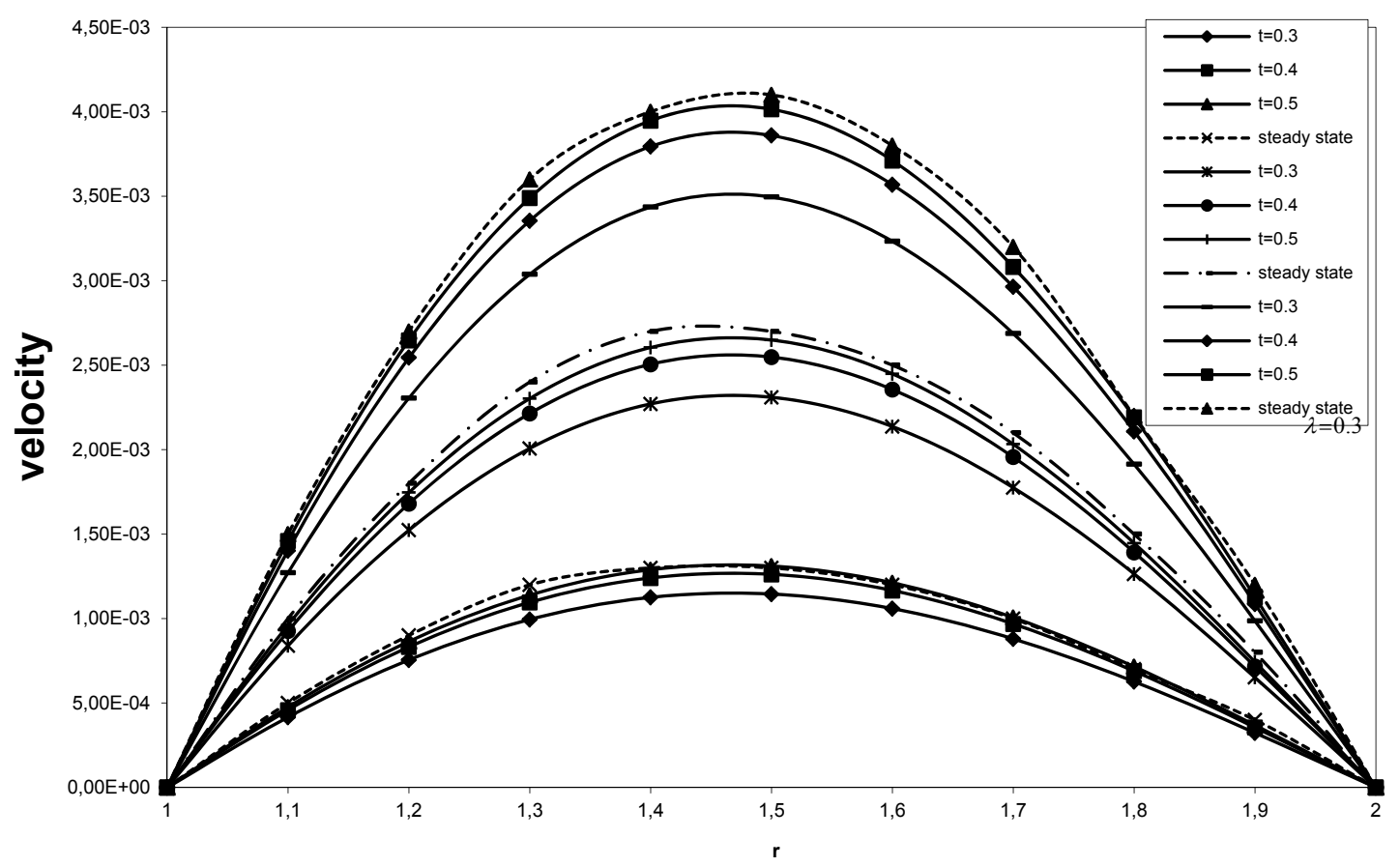

Fig.3. Velocity profile $(\varepsilon=0.01, \operatorname{Pr}=0.71)$. 


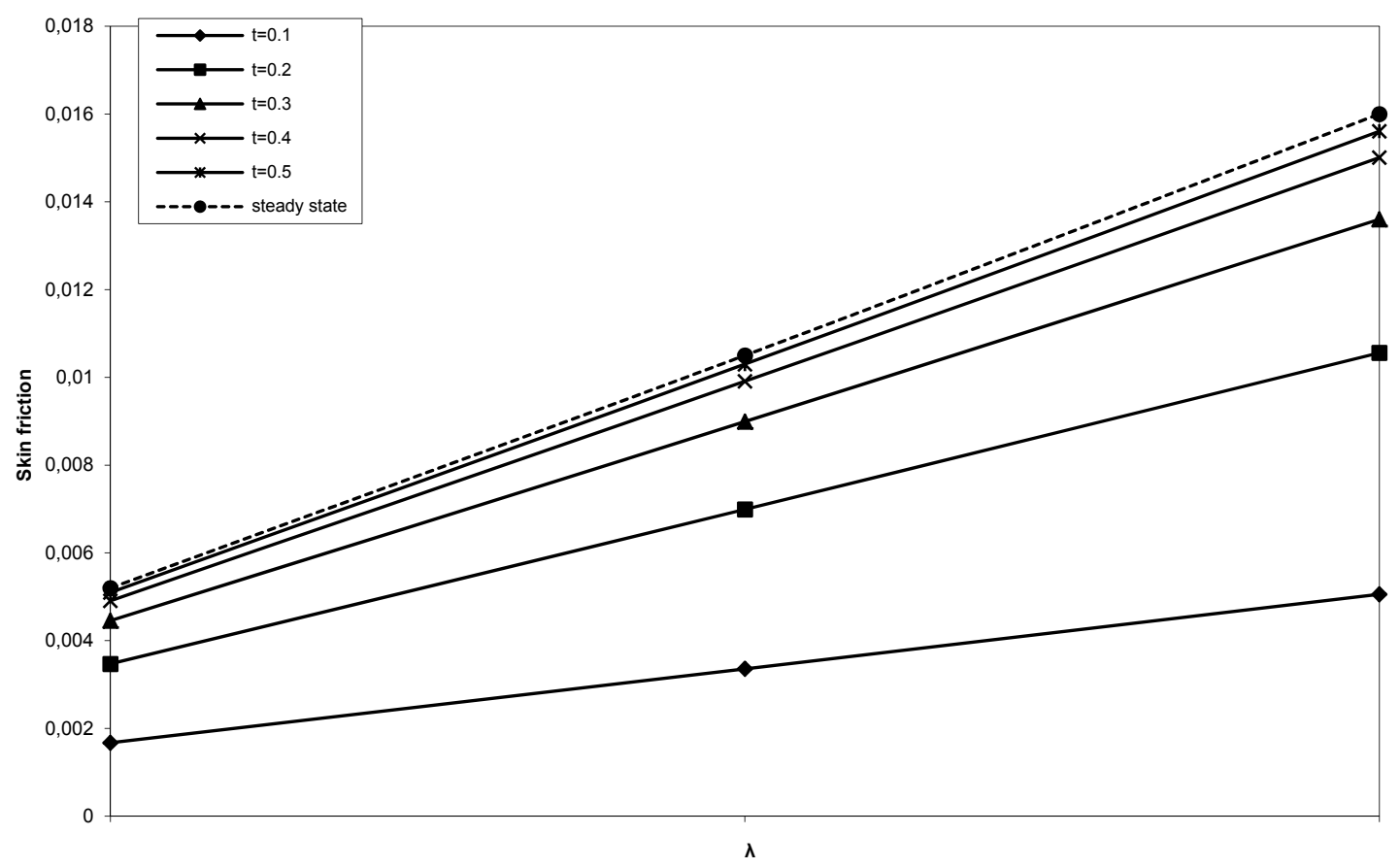

Fig.4. Skin friction at $r=1$ and $(\varepsilon=0.01, \operatorname{Pr}=0.71, \lambda=0.1,0.2,0.3)$.

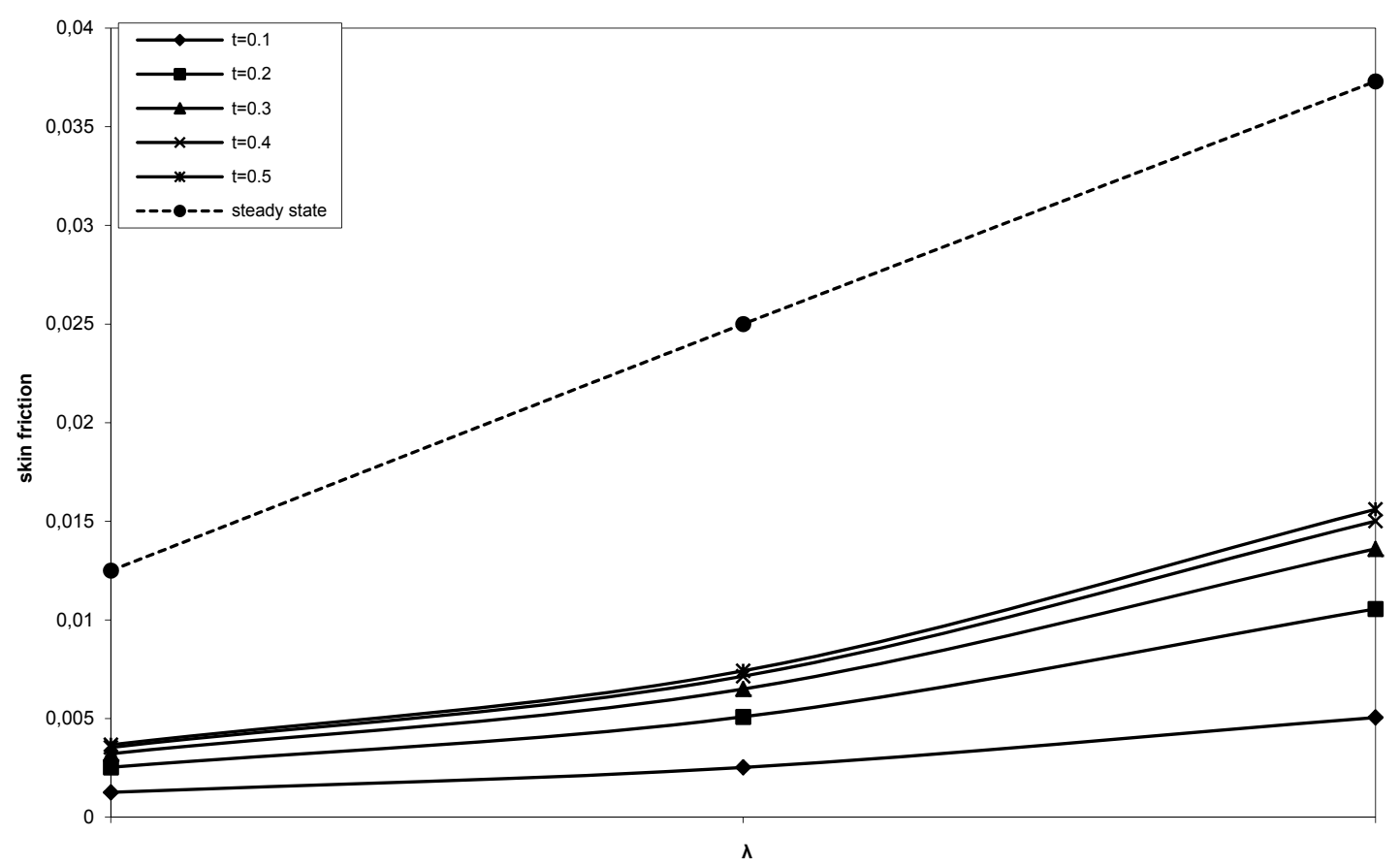

Fig.5. Skin friction at $r=b$ and $(\varepsilon=0.01, \operatorname{Pr}=0.71, \lambda=0.1,0.2,0.3)$. 


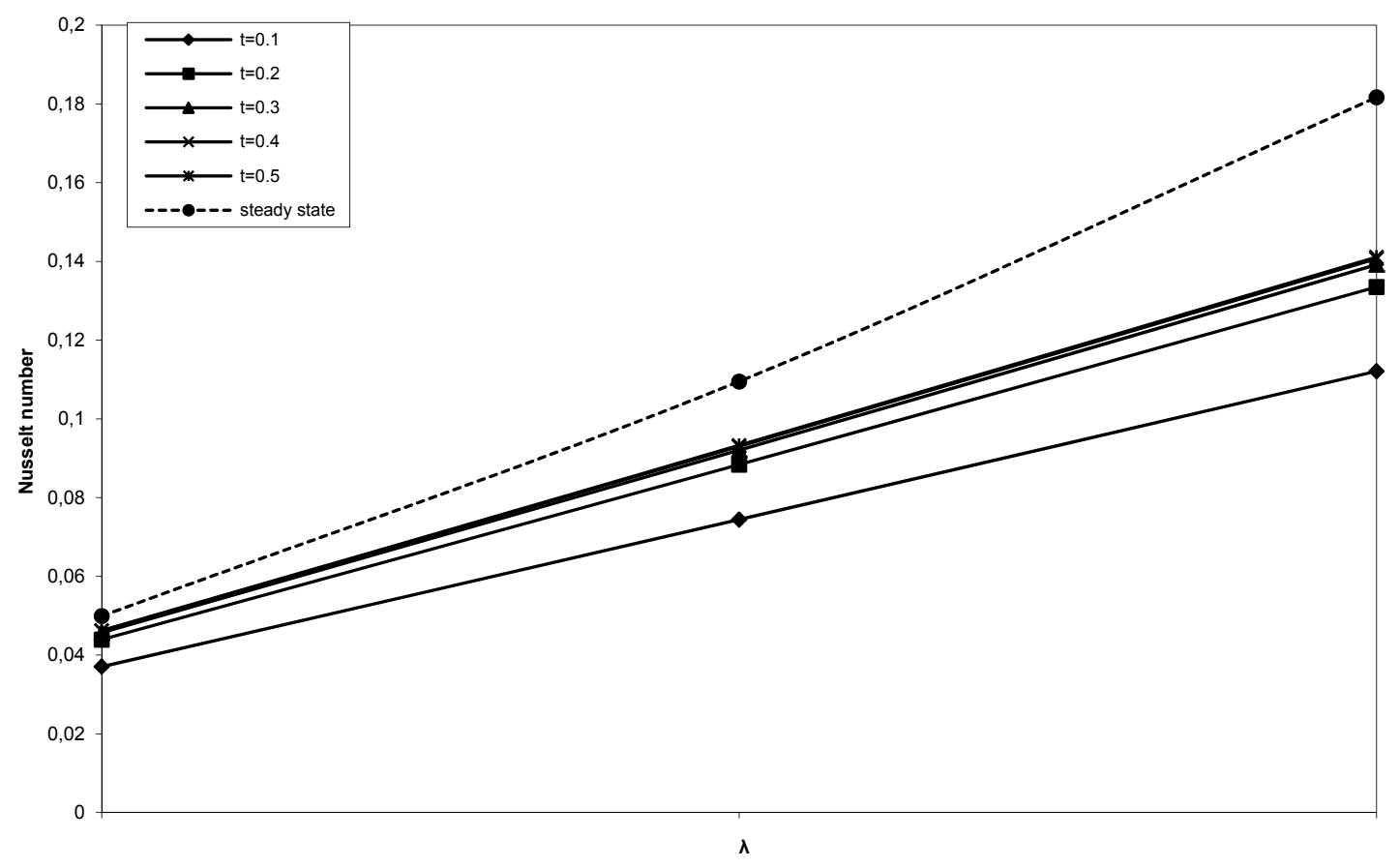

Fig.6. Nusselt number $(\mathrm{Nu})$ and $(\varepsilon=0.01, \operatorname{Pr}=0.71, \lambda=1,2,3)$.

\section{Conclusion}

Analytical expressions for velocity, temperature, skin-friction and rate of heat transfer for a steadystate fully developed natural convection flow of a viscous reactive fluid in a vertical annulus has been presented. Also time dependent non-linear problems are solved using implicit finite deference method. During the computation excellent agreement was found for the steady-state solution and numerical solution.

\section{Acknowledgement}

The author Ahmad K. Samaila is thankful to Usmanu Danfodiyo University, Sokoto for financial support.

\section{Nomenclature}

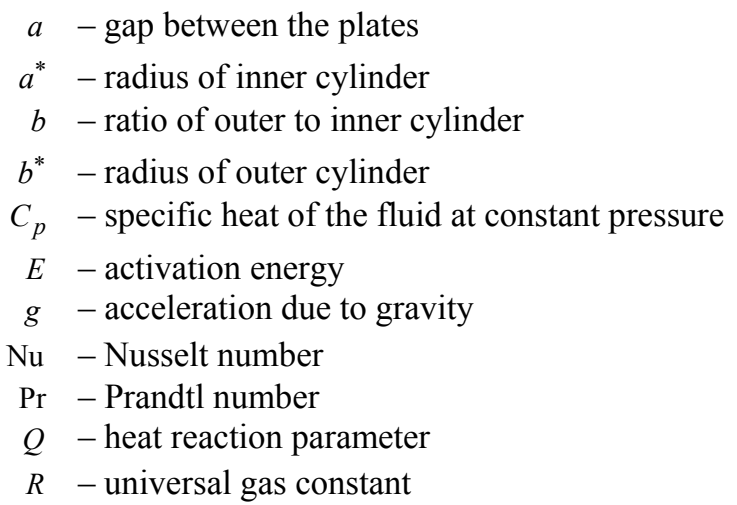




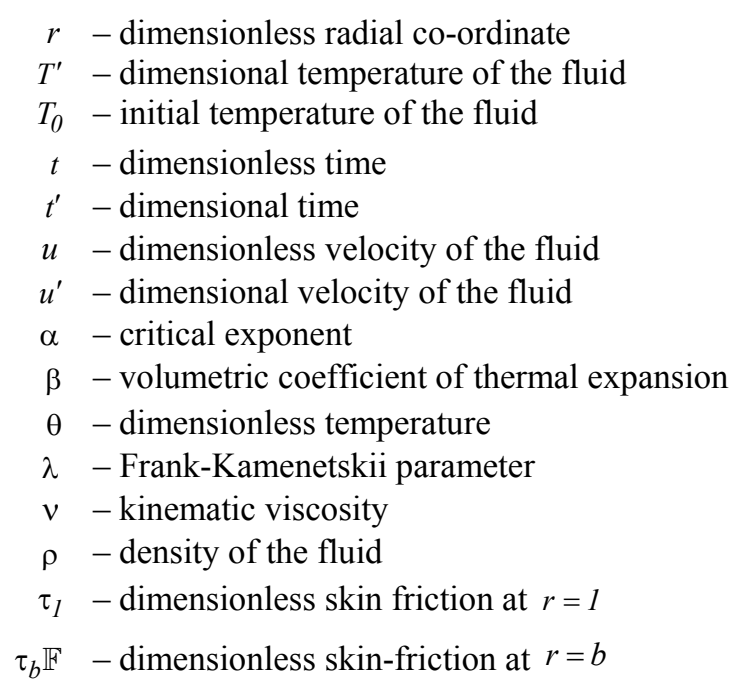

\section{References}

Ayeni R.O. (1982): On the explosion of chain-thermal reaction. - J. Aust. Math. Soc. Series, vol.24, pp.194-202.

Chao B.H., Wang H. and Cheng P. (1996): Stagnation point flow of a chemically reactive fluid in a catalytic porous bed. - International Journal of Heat and Mass Transfer, vol.39, pp.3003-3019.

Chaudary M.A. and Merkin J.H. (1994): Free-convection stagnation-point boundary layers driven by catalytic surface reactions: I The steady states. - Journal of Engineering Mathematics, vol. 28, pp.145-171.

Dainton F.S. (1960): Chain Reaction. An introduction. - New York: Willey.

El-shaarawi M.A.I. and Al-Nimr M.A. (1990): Fully developed laminar natural convection in open-ended vertical concentric annuli. - Int. J. Heat Mass Transfer, vol.9, pp.1873-1884.

El-shaarawi M.A.I. and Sarhan A. (1981): Developing laminar free convection in an open ended vertical annulus with a rotating inner cylinder. - ASME, J. Heat Transfer, vol.103, pp.552-558.

Frank Kamenetskii D.A. (1969): Diffusion and Heat Transfer in Chemical Kinetics. -New York: Plenum Press.

Hosseini R., Heyrani-Nobari M.R. and Hatam M. (2005): An experimental study of heat transfer in an open-ended vertical eccentric annulus with insulated and constant heat flux boundaries. - Applied Thermal Engineering, vol.25, No.8-9, pp.1247-1257.

Jha B.K. (2001): Transient free-convective flow in a vertical channel with heat sinks. - Int. J. Appl. Mech. and Eng. vol.6, No.2, pp.279-286.

Jha B.K., Singh A.K. and Takhar H.S. (2003): Transient free-convective flow in a vertical channel due to symmetric heating. - Int. J. Appl. Mech. Eng., vol.8, No.3, pp.497-502.

Jha B.K and Ajibade A.O. (2010): Transient natural convection flow between vertical parallel plates: one plate isothermally heated and the other thermally insulated. - J. Process Mechanical Engineering, vol.224, Part E.

Jha B.K., Samaila A.K. and Ajibade A.O. (2011): Transient free-convective flow of reactive viscous fluid in a vertical channel. - International Communication in Heat and Mass Transfer, Elsevier, vol.38, pp.633-637.

Merkin J.H. and Chaudary M.A. (1994): Free-convection boundary layers on vertical surfaces driven by an exo-thermic surface reaction. - Quarterly Journal of Mechanics and Applied Mathematics, vol.47, pp.405-428.

Makinde O.D. (2005): Strongly exo-thermic explosions in a cylindrical pipe: a case study of series summation technique. - Mechanics Research Communications, vol.32, pp.191-195.

Makinde O.D. (2006): Thermal ignition in a reactive viscous flow through a channel filled with a porous medium.A.S.M.E. J. Heat Transfer, vol.128, pp.601-604. 
Makinde O.D. (2008): Thermal criticality in viscous reactive flows through channels with a sliding wall: An exploitation of the Hermite-Pade approximation method. - Mathematical and Computer Modelling, vol.47, pp.312317.

Mint B.J., Ingham D.B. and Pop I. (1998): Free convective driven by an exothermic reaction on a vertical surface embedded in porous media. - Int. J. Heat Mass Transfer, vol.41, No.1, pp.11-23

Singh A.K., Gholami H.R. and Soundalgeker V.M. (1996): Transient free convective flow between two vertical parallel plates. - Heat Mass Transfer, vol.31, No.5, pp.329-331.

Tsao H.K. (2000): Note on electroosmotic flow through an annulus. - Journal of Colloid and Interface Science, vol.225, pp.247-250.

Yasutomi S., Blair S. and Winner W.O. (1984): An application of a free volume model to lubricant rheology. 1. Dependence of viscosity on temperature and pressure. - Trans. ASME. J. Tribol, vol.106, 291-303.

\section{APPENDIX}

$$
\begin{aligned}
& c_{1}=-\frac{1}{64 \ln (b)}\left[3-4 b^{2}+b^{4}\right]+\frac{\left(b^{2}-1\right)}{16[\ln (b)]^{2}}\left\{1+b^{2}(-1+\ln (b))\right\}, \\
& c_{2}=\frac{3}{64}-\frac{\left(b^{2}-1\right)}{16 \ln (b)}, \\
& c_{3}=-\frac{1}{4 \ln (b)}\left[\frac{b^{4}}{64}-\frac{b^{6}}{576}\right]-\frac{\left(b^{2}-1\right)}{1024[\ln (b)]^{2}}\left[4 b^{4} \ln (b)-6 b^{4}\right]+ \\
& +\frac{1}{4 \ln (b)}\left[c_{1}\left\{b^{2} \ln (b)-b^{2}\right\}+c_{2} b^{2}\right]-\frac{c_{4}}{\ln (b)}, \\
& c_{4}=-\frac{1}{256}+\frac{1}{2304}+\frac{6\left(b^{2}-1\right)}{1024 \ln (b)}-\frac{1}{4}\left[c_{1}-c_{2}\right],
\end{aligned}
$$

Received: August 10, 2011

Revised: April 25, 2012 